\title{
Optimal Couples of Rearrangement Invariant Spaces for Generalized Maximal Operators
}

\author{
Irshaad Ahmed ${ }^{1}$ and Waqas Nazeer ${ }^{2}$ \\ ${ }^{1}$ Department of Mathematics, Government College University, Faisalabad 38000, Pakistan \\ ${ }^{2}$ Department of Mathematics, Lahore Leads University, Lahore 54000, Pakistan \\ Correspondence should be addressed to Irshaad Ahmed; quantized84@yahoo.com
}

Received 19 August 2013; Accepted 11 February 2014; Published 19 March 2014

Academic Editor: Adrian Petrusel

Copyright (C) 2014 I. Ahmed and W. Nazeer. This is an open access article distributed under the Creative Commons Attribution License, which permits unrestricted use, distribution, and reproduction in any medium, provided the original work is properly cited.

The optimal couples of rearrangement invariant spaces for boundedness of a generalized maximal operator, associated with a quasiconcave function, have been characterized in terms of certain indices connected with rearrangement invariant spaces and quasiconcave functions.

\section{Introduction}

Let $L_{\text {loc }}$ be the space of all real-valued locally integrable functions on $\mathbf{R}^{n}$ with the Lebesgue measure. For any positive function $\phi$ on $(0, \infty)$, the generalized maximal operator $M_{\phi}$ is defined by

$$
M_{\phi} f(x)=\sup _{B \ni x} \frac{1}{\phi(|B|)} \int_{B}|f(y)| d y, \quad f \in L_{\mathrm{loc}},
$$

where the supremum is taken with respect to all balls $B$ containing $x$, and $|\cdot|$ denotes the Lebesgue measure.

Note that for $\phi(t)=t, M_{\phi}$ is the classical HardyLittlewood maximal operator $M$, and for $\phi(t)=t^{1-s / n}, 0<$ $s<n$, we get the fractional maximal operator $M^{s}$.

Let $S_{d}$ and $S_{t}$ be two classes of rearrangement invariant spaces (for definition, see Section 2). Take $E \in S_{d}$ and $G \in S_{t}$, and assume that $M_{\phi}: E \rightarrow G$, where the symbol $T: A \rightarrow B$ means that $T$ is bounded from $A$ into $B$. We say that $E$ is an optimal domain space in the class $S_{d}$ if $M_{\phi}: E^{\prime} \rightarrow G$ for any rearrangement invariant space $E^{\prime} \in S_{d}$, then it follows that $E^{\prime} \hookrightarrow E$, where by $A \hookrightarrow B$, we mean that $A$ is continuously embedded in $B$. We say that $G$ is an optimal target space in the class $S_{t}$ if $M_{\phi}: E \rightarrow G^{\prime}$ implies that $G \hookrightarrow G^{\prime}$ for any rearrangement invariant space $G^{\prime} \in S_{t}$. Finally, the couple $(E, G)$ is called optimal in the class $\left(S_{d}, S_{t}\right)$ if $E$ is an optimal domain space in the class $S_{d}$ and $G$ is an optimal target space in the class $S_{t}$.

The optimal couples for the fractional maximal operator have been characterized in [1]. The characterizations are based on certain conditions on the Boyd indices associated with rearrangement invariant spaces. Earlier, Jan Vybíral had considered the problem of optimal domain space for the operator $M_{\phi}$ in [2].

Our aim in this paper is to extend some of the results in [1] by means of replacing the function $t \mapsto t^{1-s / n}, 0<s<n$, by an arbitrary quasiconcave function. By a quasiconcave function $\phi$, we mean that $\phi$ is a positive function on $(0, \infty)$ such that $\phi$ is increasing and $t \mapsto \phi(t) / t$ is decreasing. To our end, we will impose certain conditions on rearrangement invariant spaces as well as on $\phi$ by means of the Boyd indices associated with them.

The paper is organized as follows. In Section 2, we give the necessary background material. In particular, we define the rearrangement invariant spaces. It is worthy of mention that our rearrangement invariant spaces are more general than those in [3]. Particularly, we do not use Fatou property and duality arguments. For convenience, we will say that a couple $(E, G)$ is admissible if $M_{\phi}: E \rightarrow G$. The admissible and optimal couples are characterized in Sections 3 and 4, respectively. 


\section{Preliminaries}

2.1. Rearrangement Invariant Spaces. Recall that the decreasing rearrangement of a measurable function $f$ on $\mathbf{R}^{n}$, denoted by $f^{*}$, is defined as

$$
f^{*}(t)=\inf \left\{\lambda>0: \mu_{f}(\lambda) \leq t\right\}, \quad t \geq 0,
$$

where $\mu_{f}$ is the distribution function of $f$ given as

$$
\mu_{f}(\lambda)=\left|\left\{x \in \mathbf{R}^{n}:|f(x)|>\lambda\right\}\right|, \quad \lambda \geq 0,
$$

(see, e.g., [3]).

Let $L$ be the space of all nonnegative locally integrable functions on $(0, \infty)$ with the Lebesgue measure. As usual, a quasinorm $\rho: L \rightarrow[0, \infty]$ satisfies the following conditions:

(i) $\rho(g)=0$ if and only if $g=0$ a.e.;

(ii) $\rho(a g)=a \rho(g), a \geq 0$;

(iii) $\rho(g+h) \leq c[\rho(g)+\rho(h)], c \geq 1$.

For each quasinorm $\rho$, there exists an equivalent quasinorm $\rho_{p}$ satisfying the triangle inequality $\rho_{p}^{p}\left(g_{1}+g_{2}\right) \leq$ $\rho_{p}^{p}\left(g_{1}\right)+\rho_{p}^{p}\left(g_{2}\right), g_{1}, g_{2} \in L$, for some $p \in(0,1]$ (see [4]). We will say that the quasinorm $\rho$ satisfies Minkovski inequality if for the equivalent quasinorm $\rho_{p}$, we have

$$
\rho_{p}^{p}\left(\sum g_{j}\right) \lesssim \sum \rho_{p}^{p}\left(g_{j}\right), \quad g_{j} \in L
$$

where the notation $a \lesssim b$ stands for the fact that $a$ is bounded above by a multiple of $b$, the multiple being independent of any variables in $a$ and $b$. Later we will use $a \approx b$ to indicate that both $a \leqslant b$ and $b \leqslant a$ hold.

A quasinorm $\rho$ is said to be monotone if for $g_{1}, g_{2} \in L$ with $g_{1} \leq g_{2}$, we have $\rho\left(g_{1}\right) \leq \rho\left(g_{2}\right)$.

Let $\rho_{E}: L \rightarrow[0, \infty]$ be a quasinorm, and let $E$ be the space consisting of all functions $f$ in $L_{\text {loc }}$ for which the quasinorm $\|f\|_{E}:=\rho_{E}\left(f^{*}\right)$ is finite. The space $E$ is a rearrangement invariant in the sense that if $f \in E$ and $f^{*}=g^{*}$ for $g \in L_{\text {loc }}$, then $g \in E$. We will say that $E$ is the rearrangement invariant space generated by the quasinorm $\rho_{E}$.

The rearrangement invariant Banach function spaces considered in [3] are a particular example of our rearrangement invariant spaces according to the Luxemburg representation theorem (see [3, page 62]). More general examples are given by the Riesz-Fischer monotone spaces as in [3, page 305$]$.

2.2. Boyd Indices. Let $E$ be a rearrangement invariant space generated by a quasinorm $\rho_{E}$. The lower and upper Boyd indices of $E$, denoted by $\alpha_{E}$ and $\beta_{E}$, are defined similarly to [5]. Let

$$
h_{E}(u)=\sup \left\{\frac{\rho_{E}\left(g_{u}^{*}\right)}{\rho_{E}\left(g^{*}\right)}: g \in L\right\}, \quad g_{u}(t):=g\left(\frac{t}{u}\right)
$$

be the dilation function generated by $\rho_{E}$. Suppose that $h_{E}$ is finite. Then,

$$
\alpha_{E}:=\sup _{0<t<1} \frac{\log h_{E}(t)}{\log t}, \quad \beta_{E}:=\inf _{1<t<\infty} \frac{\log h_{E}(t)}{\log t} .
$$

If $\rho_{E}$ is monotone, then the function $h_{E}$ is increasing. Hence, in view of $h_{E}(1)=1$ and $h_{E}(u) h_{E}(1 / u) \geq 1$, we obtain $0 \leq \alpha_{E} \leq \beta_{E}$. Furthermore, the submultiplicativity of $h_{E}$ will imply (see [6, page 244]) that both indices are finite and given by the following limits:

$$
\alpha_{E}=\lim _{t \rightarrow 0^{+}} \frac{\log h_{E}(t)}{\log t}, \quad \beta_{E}=\lim _{t \rightarrow \infty} \frac{\log h_{E}(t)}{\log t} .
$$

Similarly, we can associate a pair of indices with a positive function $\phi$ on $(0, \infty)$ (see [7]). In this paper, we will need only one of them which we call as the lower Boyd index $\alpha_{\phi}$. It is defined by

$$
\alpha_{\phi}=\sup _{0<t<1} \frac{\ln h_{\phi}(t)}{\ln t}
$$

where

$$
h_{\phi}(u)=\sup _{0<t<\infty} \frac{\phi(t u)}{\phi(t)}
$$

is the dilation function $h_{\phi}$ generated by $\phi$. If the function $\phi$ is quasiconcave, then $0 \leq \alpha_{\phi} \leq 1$ (see [7, page 54]).

We will make use of the assertions of the following proposition (cf. [3, Lemma 5.9, page 147]) in the forthcoming sections.

Proposition 1. Let $E$ be a rearrangement invariant space generated by a monotone quasinorm $\rho_{E}$, and let $\phi$ be a quasiconcave function. Then, for any $\alpha>0$,

$$
\begin{gathered}
\int_{0}^{1} v^{-\alpha} h_{E}^{\alpha}(v) h_{\phi}^{\alpha}(v) \frac{d v}{v}<\infty, \quad \text { if } \alpha_{E}+\alpha_{\phi}>1, \\
\int_{0}^{1} h_{E}^{\alpha}\left(\frac{1}{v}\right) h_{\phi}^{\alpha}(v) \frac{d v}{v}<\infty, \quad \text { if } \beta_{E}<\alpha_{\phi} .
\end{gathered}
$$

Proof. We will derive only (10); the proof of (11) will be similar. Let $\epsilon$ be small enough such that $\alpha_{E}+\alpha_{\phi}-2 \epsilon>1$. For this $\epsilon$, there exists $0<T_{1}<1$ such that

$$
h_{E}(t)<t^{\alpha_{E}-\epsilon}, \quad 0<t \leq T_{1},
$$

and there also exists $0<T_{2}<1$ such that

$$
h_{\phi}(t)<t^{\alpha_{\phi}-\epsilon}, \quad 0<t \leq T_{2} .
$$

Taking $T=\min \left(T_{1}, T_{2}\right)$, we get

$$
h_{E}(t) h_{\phi}(t)<t^{\alpha_{E}+\alpha_{\phi}-2 \epsilon}, \quad 0<t \leq T,
$$

thus

$$
\begin{aligned}
& \int_{0}^{1} v^{-\alpha} h_{E}^{\alpha}(v) h_{\phi}^{\alpha}(v) \frac{d v}{v} \\
& \quad<\int_{0}^{T} v^{\alpha\left(-1+\alpha_{E}+\alpha_{\phi}-2 \epsilon\right)} \frac{d v}{v}+\int_{T}^{1} v^{-\alpha} h_{E}^{\alpha}(v) h_{\phi}^{\alpha}(v) \frac{d v}{v} .
\end{aligned}
$$

We note that first integral on right hand side is convergent since $\alpha_{E}+\alpha_{\phi}-2 \epsilon>1$. The second integral is also convergent since it is estimated from above by a convergent integral $\int_{T}^{1} v^{-\alpha}(d v / v)$; this completes the proof. 


\section{Admissible Couples}

In what follows, $\phi$ will be a fixed quasiconcave function. We will need to work with the following classes of rearrangement invariant spaces:

$S_{0}$ consists of all rearrangement invariant spaces $E$ generated by a monotone quasinorm $\rho_{E}$;

$S_{d, 1}$ consists of all rearrangement invariant spaces $E$ generated by a quasinorm $\rho_{E}$ which is monotone and satisfies Minkovski inequality along with $\alpha_{E}+\alpha_{\phi}>1$.

In the formulation of our results, we will need also a subclass $L_{0}$ of $L$, formed of all decreasing functions in $L$.

We note here that the admissibility of a couple $(E, G)$ (i.e., $\left.M_{\phi}: E \rightarrow G\right)$ is equivalent to the following estimate:

$$
\rho_{G}\left(\left(M_{\phi} f\right)^{*}\right) \lesssim \rho_{E}\left(f^{*}\right), \quad f \in E
$$

Our starting point is the following characterization of all admissible couples which is essentially a reformulation of the sharp rearrangement inequality which was proved in $[2$, Theorem 3.13].

Theorem 2. Let $E, G \in S_{0}$; then the couple $(E, G)$ is admissible if and only if

$$
\rho_{G}(N g) \lesssim \rho_{E}(g), \quad g \in L_{0}
$$

where

$$
N g(t):=\sup _{u \geq t} \frac{1}{\phi(u)} \int_{0}^{u} g(u) d u, \quad t>0 .
$$

The one-dimensional operator $N$ is rather complicated; however, we can replace it by a simpler one in the condition (17) by exploiting the next estimate.

Lemma 3. Assume that $E \in S_{d, 1}$. Then,

$$
\rho_{E}\left(t^{-1} \phi(t) Q g(t)\right) \lesssim \rho_{E}\left(t^{-1} \phi(t) g(t)\right), \quad g \in L_{0},
$$

where

$$
Q g(t):=\int_{t}^{\infty} g(s) \frac{d s}{s}, \quad t>0
$$

Proof. We start off with a simple change of variables to have

$$
Q g(t)=\int_{0}^{1} g\left(\frac{t}{v}\right) \frac{d v}{v}
$$

Since the function $v \mapsto g(t / v)$ is increasing in $v$, therefore

$$
\begin{aligned}
Q g(t) & =\sum_{k=-\infty}^{-1} \int_{2^{k}}^{2^{k+1}} g\left(\frac{t}{v}\right) \frac{d v}{v} \\
& \leq \sum_{k=-\infty}^{-1} g\left(\frac{t}{2^{k+1}}\right) \int_{2^{k}}^{2^{k+1}} \frac{d v}{v} \\
& \approx \sum_{k=-\infty}^{-1} g\left(\frac{t}{2^{k+1}}\right) .
\end{aligned}
$$

As $\rho_{E}$ is monotone and satisfies Minkowski's inequality, we get

$$
\rho_{E}^{p}\left(t^{-1} \phi(t) Q g(t)\right) \lesssim \sum_{k=-\infty}^{-1} \rho_{E}^{p}\left(t^{-1} \phi(t) g\left(\frac{t}{2^{k+1}}\right)\right) .
$$

Now we observe that the function $v \mapsto \rho_{E}^{p}\left(t^{-1} \phi(t) g(t / 2 v)\right)$ is increasing in $v$; thus,

$$
\begin{aligned}
& \rho_{E}^{p}\left(t^{-1} \phi(t) Q g(t)\right) \\
& \quad \lesssim \sum_{k=-\infty}^{-1} \int_{2^{k}}^{2^{k+1}} \rho_{E}^{p}\left(t^{-1} \phi(t) g\left(\frac{t}{2 v}\right)\right) \frac{d v}{v} \\
& \quad \lesssim \int_{0}^{1} \rho_{E}^{p}\left(t^{-1} \phi(t) g\left(\frac{t}{2 v}\right)\right) \frac{d v}{v}
\end{aligned}
$$

Finally, using the definitions of $h_{\phi}$ and $h_{E}$ along with the quasiconcavity of $\phi$, we obtain

$$
\begin{aligned}
\rho_{E}^{p} & \left(t^{-1} \phi(t) Q g(t)\right) \\
& \lesssim \int_{0}^{1} h_{\phi}^{p}(v) \rho_{E}^{p}\left(t^{-1} \phi\left(\frac{t}{v}\right) g\left(\frac{t}{2 v}\right)\right) \frac{d v}{v} \\
& \approx \int_{0}^{1} h_{\phi}^{p}(v) \rho_{E}^{p}\left(t^{-1} \phi\left(\frac{t}{2 v}\right) g\left(\frac{t}{2 v}\right)\right) \frac{d v}{v} \\
& \lesssim \rho_{E}^{p}\left(t^{-1} \phi(t) g(t)\right) \int_{0}^{1} h_{\phi}^{p}(2 v) v^{-p} h_{E}^{p}(2 v) \frac{d v}{v} \\
& \approx \rho_{E}^{p}\left(t^{-1} \phi(t) g(t)\right) \int_{0}^{2} v^{-p} h_{\phi}^{p}(v) h_{E}^{p}(v) \frac{d v}{v},
\end{aligned}
$$

and the required estimate follows thanks to (10).

Theorem 4. Let $E \in S_{d, 1}$ and $G \in S_{0}$. Then, the couple $(E, G)$ is admissible if and only if

$$
\rho_{G}(T g) \lesssim \rho_{E}(g), \quad g \in L_{0},
$$

where

$$
\operatorname{Tg}(t):=\frac{1}{\phi(t)} \int_{0}^{t} g(u) d u, \quad t>0
$$

Remark 5. Note that if $\phi$ is an identity function, then the condition $\alpha_{E}+\alpha_{\phi}>1$, which turns into $\alpha_{E}>0$, is not needed since $\mathrm{Ng}=\mathrm{Tg}$ if $g \in L_{0}$.

Proof. Assume first that the couple $(E, G)$ is admissible. Then, in view of the condition (17), $T \leq N$, and the monotonicity of $\rho_{G}$, the condition (26) follows immediately.

Conversely, assume that the condition (26) holds, and fix $g \in L_{0}$. Denoting

$$
T^{\prime} g(t):=\sup _{u \geq t} \frac{1}{\phi(u)} \int_{t}^{u} g(v) d v
$$

we note that $N \approx T+T^{\prime}$ as $\phi$ is increasing. Then, by Theorem 2, it will suffice to establish that $\rho_{G}\left(T^{\prime} g\right) \lesssim \rho_{E}(g)$. 
Since both the functions $t \mapsto t^{-1} \phi(t)$ and $t \mapsto T^{\prime} g(t)$ are decreasing in $t$, we have

$$
T^{\prime} g \leq T g_{1}
$$

where $g_{1}(t):=t^{-1} \phi(t) T^{\prime} g(t)$. Observe that $g_{1} \in L_{0}$. Therefore, using the monotonicity of $\rho_{G}$ and applying the condition (26), we obtain $\rho_{G}\left(T^{\prime} g\right) \lesssim \rho_{E}\left(g_{1}\right)$. The proof will thus be complete if we show that $\rho_{E}\left(g_{1}\right) \lesssim \rho_{E}(g)$. To this end, we set $g_{2}(t):=\operatorname{tg}(t) / \phi(t)$. Then, by definition of $Q$, we have

$$
Q g_{2}(t) \geq \int_{t}^{u} \frac{g(v)}{\phi(v)} d v
$$

whenever $u \geq t$. As $\phi$ is increasing, we further have

$$
Q g_{2}(t) \geq \frac{1}{\phi(v)} \int_{t}^{u} g(v) d v
$$

whenever $u \geq t$, whence we get

$$
Q g_{2}(t) \geq T^{\prime} g(t)
$$

by taking supremum over all $u \geq t$. Finally, multiplying both sides by $t^{-1} \phi(t)$, using monotonicity of $\rho_{E}$, and applying Lemma 3, we arrive at

$$
\rho_{E}\left(g_{1}\right) \lesssim \rho_{E}(g),
$$

as desired. The proof is complete.

Remark 6. By Theorem 1.3 in [8], the conditions (17) and (26) are also equivalent if $G$ is a rearrangement invariant space as in [3]. The key ingredient of the proof of the above mentioned theorem is the Hardy-Littlewood-Póyla principle (see [3, Theorem 4.6, page 61]) which is not guaranteed in our rearrangement invariant spaces.

\section{Optimal Couples}

We introduce two more classes of rearrangement invariant spaces as follows:

$S_{d, 2}$ consists of all rearrangement invariant spaces $E$ generated by a quasinorm $\rho_{E}$ which is monotone and satisfies Minkovski inequality along with $\alpha_{E}+\alpha_{\phi}>1$ and $\beta_{E}<1$;

$S_{t, 1}$ consists of all rearrangement invariant spaces $G$ generated by a quasinorm $\rho_{G}$ which is monotone and satisfies Minkovski inequality along with $\beta_{G}<\alpha_{\phi}$.

First we construct optimal couples with the aid of Theorem 2. We will need the next estimate which can be proved by using Minkovski inequality and (11) as in Lemma 3.

Lemma 7. Let $G \in S_{t, 1}$; then,

$$
\rho_{G}\left(\frac{t}{\phi(t)} P g(t)\right) \lesssim \rho_{G}\left(\frac{t}{\phi(t)} g(t)\right), \quad g \in L_{0},
$$

where

$$
P g(t):=\frac{1}{t} \int_{0}^{t} g(s) d s, \quad t>0
$$

Remark 8. In fact, the above estimate is two sided because the reverse estimate holds trivially since $P g \geq g$ if $g \in L_{0}$.

Theorem 9. Let $G \in S_{t, 1}$ be a given rearrangement invariant space. Let $E$ be the rearrangement invariant space generated by the quasinorm as follows:

$$
\rho_{E}(g)=\rho_{G}\left(N g^{*}\right), \quad g \in L .
$$

Then, the couple $(E, G)$ is optimal in the class $\left(S_{0}, S_{t, 1}\right)$.

Proof. Clearly, the couple $(E, G)$ is admissible by Theorem 2 . To see that $E$ is the optimal domain space in the class $S_{0}$, let $E^{\prime} \in S_{0}$ be another rearrangement invariant space such that the couple $\left(E^{\prime}, G\right)$ is admissible. Take $f \in E^{\prime}$ and apply Theorem 2 to obtain

$$
\rho_{E}\left(f^{*}\right)=\rho_{G}\left(N f^{*}\right) \lesssim \rho_{E^{\prime}}\left(f^{*}\right)
$$

where we get $E^{\prime} \hookrightarrow E$ as desired.

It now remains to establish that $G$ is the optimal target space in the class $S_{t, 1}$. Let $G^{\prime} \in S_{t, 1}$ be another rearrangement space such that the couple $\left(E, G^{\prime}\right)$ is admissible, and take $f \in$ G. Set

$$
f_{1}(t):=t^{-1} \phi(t) f^{*}(t),
$$

to observe that $f^{*} \leq N f_{1}$ because $f_{1} \in L_{0}$. Consequently,

$$
\begin{aligned}
\rho_{G^{\prime}}\left(f^{*}\right) & \leq \rho_{G^{\prime}}\left(N f_{1}\right) \\
& \leq \rho_{E}\left(f_{1}\right),
\end{aligned}
$$

again by Theorem 2 . It follows, by definition of $\rho_{E}$, that $\rho_{G^{\prime}}\left(f^{*}\right) \lesssim \rho_{G}\left(N f_{1}\right)$. In view of $N \approx T+T^{\prime}$, we can write the following:

$$
\rho_{G^{\prime}}\left(f^{*}\right) \lesssim \rho_{G}\left(T f_{1}\right)+\rho_{G}\left(T^{\prime} f_{1}\right) .
$$

Since $T f_{1}(t)=(t / \phi(t)) P f_{1}(t)$, an application of Lemma 7 yields

$$
\rho_{G^{\prime}}\left(f^{*}\right) \lesssim \rho_{G}\left(f^{*}\right)+\rho_{G}\left(T^{\prime} f_{1}\right) .
$$

Hence, the required embedding $G \hookrightarrow G^{\prime}$ will follow if we show that $\rho_{G}\left(T^{\prime} f_{1}\right) \lesssim \rho_{G}\left(f^{*}\right)$. For this, let us note that

$$
\begin{aligned}
T^{\prime} f_{1}(t) & =\sup _{u \geq t} \frac{1}{\phi(u)} \int_{t}^{u} \phi(v) f^{*}(v) \frac{d v}{v} \\
& \leq f^{*}(t) \sup _{u \geq t} \frac{1}{\phi(u)} \int_{t}^{u} \phi(v) \frac{d v}{v} \\
& \leq f^{*}(t) \sup _{u \geq t} \frac{1}{\phi(u)} \int_{0}^{u} \phi(v) \frac{d v}{v} .
\end{aligned}
$$

Since $0 \leq \beta_{G}<\alpha_{\phi}$, thus $\alpha_{\phi}>0$. Therefore, by [7, Corollary 3, page 57], we get $T^{\prime} f_{1} \lesssim f^{*}$. This gives $\rho_{G^{\prime}}\left(T^{\prime} f_{1}\right) \lesssim \rho_{G}\left(f^{*}\right)$ as desired. The proof is complete.

Now we apply Theorem 4 to construct optimal couples. 
Theorem 10. Let $E \in S_{d, 2}$ be a given rearrangement invariant space. Let $G$ be the rearrangement invariant space generated by the quasinorm as follows:

$$
\rho_{G}(g)=\rho_{E}\left(t^{-1} \phi(t) g(t)\right), \quad g \in L .
$$

Then, the couple $(E, G)$ is optimal in the class $\left(S_{d, 2}, S_{0}\right)$.

Proof. Let $g \in L_{0}$. Then, by the definition of $\rho_{G}$,

$$
\rho_{G}(T g)=\rho_{E}\left(\frac{1}{t} \int_{0}^{t} g(v) d v\right)
$$

Since $\beta_{E}<1$, we can apply Lemma 7 to $\phi(t)=t$ to obtain $\rho_{G}(\mathrm{Tg}) \lesssim \rho_{E}(g)$. Hence, the couple $(E, G)$ is admissible by Theorem 4 .

To see that $G$ is the optimal target space in the class $S_{0}$, let $G^{\prime} \in S_{0}$ be another rearrangement space such that the couple $\left(E, G^{\prime}\right)$ is admissible. Given that $f \in G$, consider again the function $f_{1}(t)=t^{-1} \phi(t) f^{*}(t) \in L_{0}$. Then, $f^{*} \leq T f_{1}$. Thus,

$$
\rho_{G^{\prime}}\left(f^{*}\right) \leq \rho_{G^{\prime}}\left(T f_{1}\right) \lesssim \rho_{E}\left(f_{1}\right),
$$

by Theorem 4 . Therefore,

$$
\rho_{G^{\prime}}\left(f^{*}\right) \lesssim \rho_{E}\left(t^{-1} \phi(t) f^{*}(t)\right)=\rho_{G}\left(f^{*}\right),
$$

whence we get the desired embedding $G \hookrightarrow G^{\prime}$.

Our remaining task is to show that $E$ is the optimal domain space in the class $S_{d, 2}$. To do so, let $E^{\prime} \in S_{d, 2}$ be another rearrangement invariant space such that the couple $\left(E^{\prime}, G\right)$ is admissible. Take $f \in E^{\prime}$ and note that $f^{*}(t) \leq$ $t^{-1} \phi(t) T f^{*}(t)$. Thus,

$$
\rho_{E}\left(f^{*}\right) \leq \rho_{E}\left(t^{-1} \phi(t) T f^{*}\right)=\rho_{G}\left(T f^{*}\right),
$$

which gives $\rho_{E}\left(f^{*}\right) \lesssim \rho_{E^{\prime}}\left(f^{*}\right)$ by Theorem 4 . It follows that $E^{\prime} \hookrightarrow E$ as required. The proof is complete.

Remark 11. The statements of Theorems 9 and 10 generalize those of Theorems 2.10 and 3.1 in [1], respectively.

\section{Conflict of Interests}

The authors declare that there is no conflict of interests regarding the publication of this paper.

\section{References}

[1] I. Ahmed, G. Karadzhov, and W. Nazeer, "Optimal couples of rearrangement invariant spaces for the fractional maximal operator,' Comptes Rendus de l'Académie Bulgare des Sciences, vol. 64, no. 9, pp. 1233-1240, 2011.

[2] J. Vybíral, Optimality of Function Spaces for Boundedness of Integral Operators and Sobolev Embeddings, Faculty of Mathematics and Physics, Charles University, Prague, Czech Republic, 2002.

[3] C. Bennett and R. Sharpley, Interpolation of Operators, vol. 129, Academic Press, Boston, Mass, USA, 1988.

[4] G. Köthe, Topologisch Lineare Räume. I, Springer, Berlin, Germany, 1966.
[5] D. W. Boyd, "Indices of function spaces and their relationship to interpolation," Canadian Journal of Mathematics, vol. 21, pp. 1245-1254, 1969.

[6] E. Hille and R. S. Phillips, Functional Analysis and Semi-Groups, American Mathematical Society, Providence, RI, USA, 1957.

[7] S. G. Krein, Ju. I. Petunin, and E. M. Semenov, Interpolation of Linear Operators, vol. 54, American Mathematical Society, Providence, RI, USA, 1982.

[8] L. Malý, "Calderón-type theorems for operators with nonstandard endpoint behavior on Lorentz spaces," Mathematische Nachrichten, vol. 285, no. 11-12, pp. 1450-1465, 2012. 


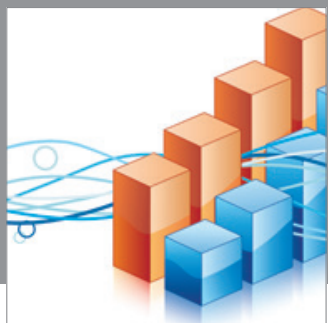

Advances in

Operations Research

mansans

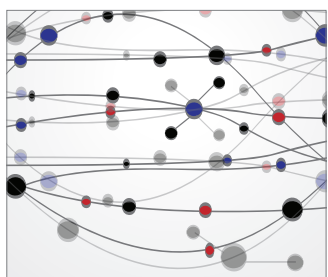

The Scientific World Journal
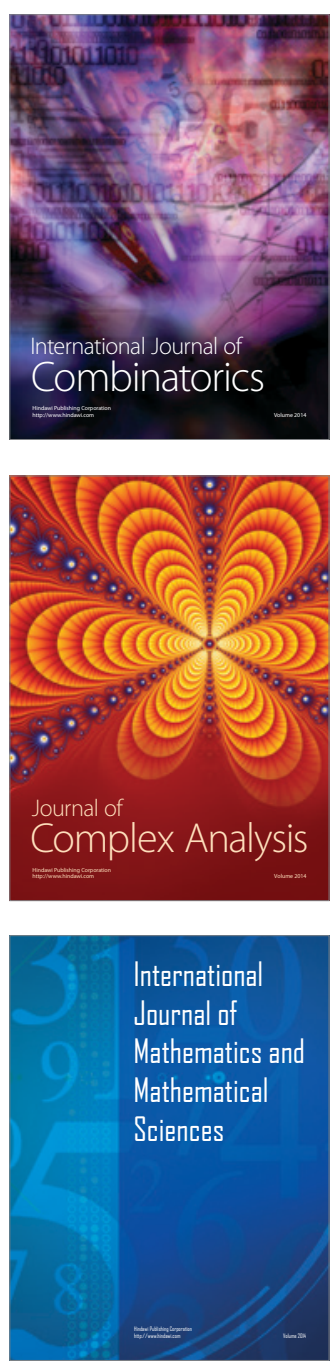
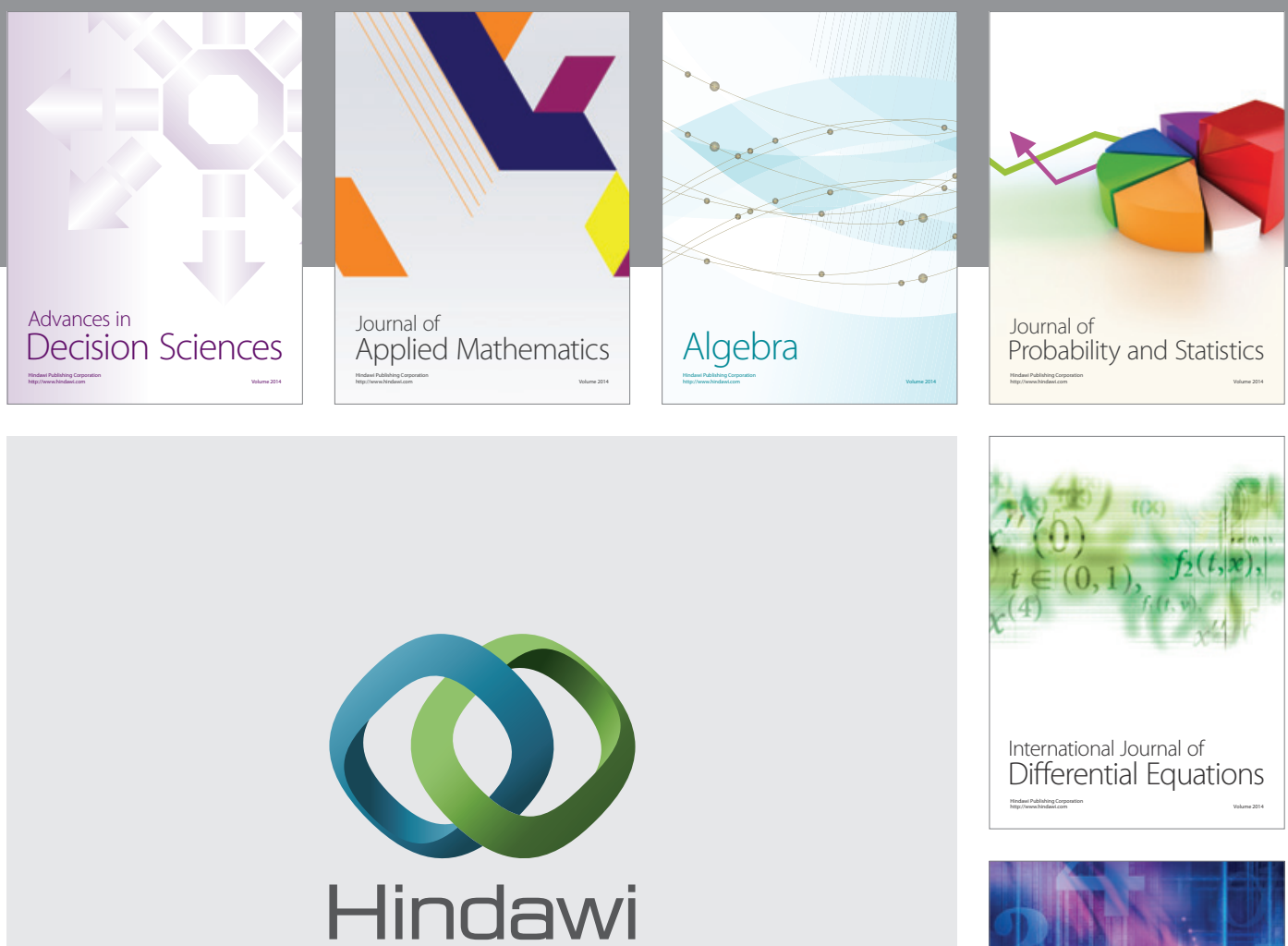

Submit your manuscripts at http://www.hindawi.com
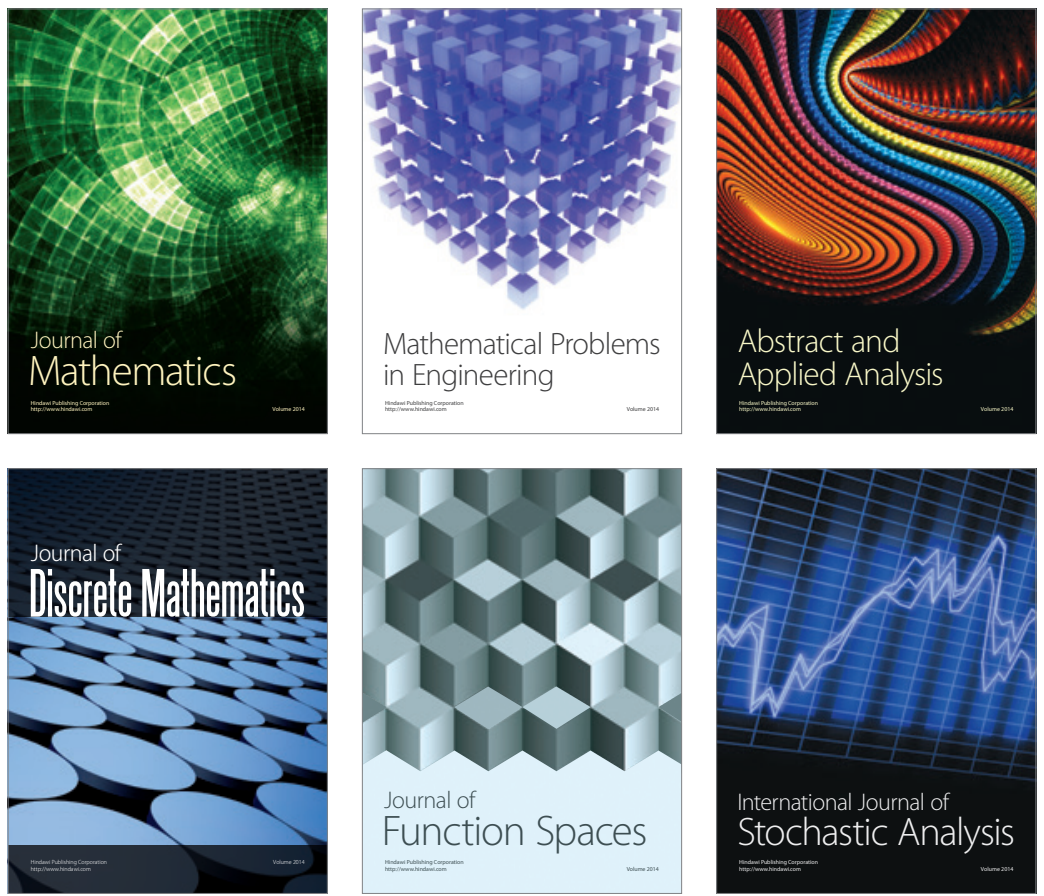

Journal of

Function Spaces

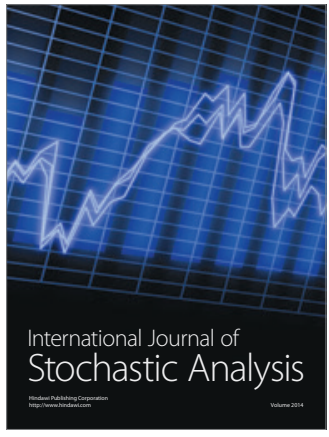

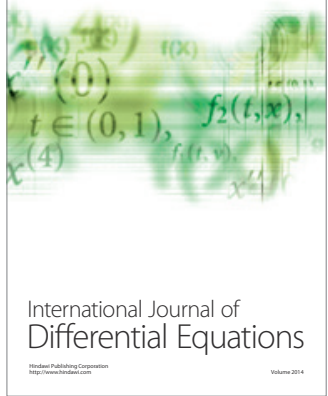
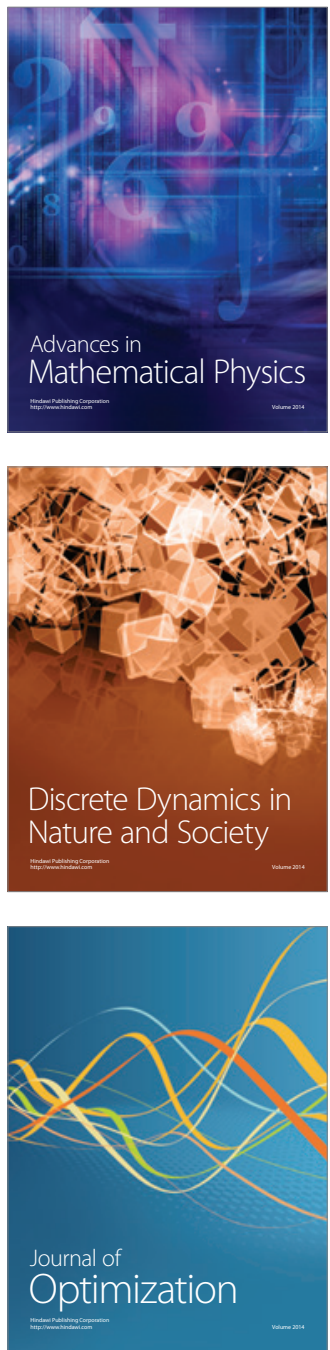